\title{
Les catégories d'emploi dans la société japonaise
}

Employment Categories in Japanese Society

\section{Kurumi Sugita}

\section{CpenEdition}

\section{Journals}

Édition électronique

URL : http://journals.openedition.org/travailemploi/4844

DOI : 10.4000/travailemploi.4844

ISSN : 1775-416X

\section{Éditeur}

DARES - Ministère du Travail

\section{Édition imprimée}

Date de publication : 15 octobre 2006

Pagination : 19-32

ISSN : 0224-4365

\section{Référence électronique}

Kurumi Sugita, «Les catégories d'emploi dans la société japonaise », Travail et Emploi [En ligne],

108 | octobre-décembre 2006, mis en ligne le 15 décembre 2008, consulté le 03 mai 2019. URL http://journals.openedition.org/travailemploi/4844; DOI : 10.4000/travailemploi.4844 


\title{
Les catégories d'emploi dans la société japonaise
}

\author{
Kurumi Sugita $(*)$
}

\begin{abstract}
Les praticiens des comparaisons internationales en sciences sociales sont confrontés à la question de la comparabilité de leurs outils d'analyse, et notamment des catégories statistiques ou conceptuelles qu'ils emploient. L'auteure traite ici des catégories d'emploi telles qu'elles sont utilisées dans les enquêtes sur l'emploi et le travail au Japon. Elle part d'un examen minutieux des questionnaires de différentes enquêtes sur l'emploi pour observer les divers usages qui sont faits de ces catégories, dans une double perspective, historique (examen des variations de sens au cours du temps) et transversale (variations d'une enquête à l'autre). L'usage de ces catégories nécessite beaucoup de précautions, et doit tenir compte des relations existant entre elles, et les resituer dans leur contexte historique. L'auteure met en évidence le flou qui entoure désormais certaines catégories du fait de l'effacement progressif de leur signification lié à la sédimentation des enquêtes. Elle en souligne le caractère équivoque, du fait en particulier du non-recouvrement entre des stratifications de significations successives, ce qui a rendu flou le contour de certaines d'entre elles. L'article donne plusieurs interprétations possibles de leur caractère équivoque. Par exemple, le fait que la dénomination usuelle et quotidienne des différentes formes d'emploi soit également utilisée par le système d'observation statistique. Ou le fait encore que le système de classification savant se base sur les pratiques successives des entreprises, témoignant de leur importance dans la société japonaise. En tant que constructions sociales, les catégories du système statistique sont porteuses des évolutions de la société, incorporant au fil du temps des significations suivant des logiques différentes, qui contribuent à les rendre ambiguës, et peuvent prêter à confusion. Un glossaire en annexe aide à démêler ces imbrications de sens.
\end{abstract}

L'objet de cet article est d'analyser les catégories d'emploi telles qu'elles sont utilisées actuellement dans le système statistique japonais, et de rendre compte de la cohérence et des tensions qui existent entre elles. Les catégories d'emploi sont considérées ici comme des constructions sociales interdépendantes, se définissant les unes par rapport aux autres. Ce sont les relations entre elles, et la configuration de l'ensemble de ces catégories qui nous intéresseront. Dire que les catégories d'emploi sont des constructions sociales signifie qu'elles peuvent changer au fil du temps. Je vais donc les examiner dans leur dimension historique, sans toutefois m'engager dans l'étude de leur première mise en place. Le présent article se limitera à l'examen de leur évolution, et tendra à mettre en évidence les ressorts sociaux des définitions produites au fil du temps à la fois par les institutions statistiques, par les entreprises et par les individus eux-mêmes dans les réponses qu'ils font aux enquêtes.

Pour ce faire, je m'appuierai sur l'étude des questionnaires de quelques enquêtes publiques concernant la force de travail. Je n'aborderai pas ici la question de la qualité des réponses, fondées sur l'auto-évaluation, ni la nécessité de les replacer

\footnotetext{
* Chercheuse à l'Institut d'Asie Orientale, ENS LHS Lyon; kurumi.sugita@ens-1sh.fr
}

dans leur contexte. J'analyserai les questionnaires des enquêtes nationales et tout spécialement leur terminologie. J'utiliserai comme corpus de données la traduction officielle en anglais fournie dans les rapports d'enquêtes, car cette traduction est souvent révélatrice de la complexité latente des catégories(1). Je ne traiterai pas ici des catégories juridiques, pourtant étroitement imbriquées avec les catégories statistiques, l'articulation dynamique entre ces deux champs méritant une analyse ultérieure (2). L'article commencera par une esquisse de l'histoire des entreprises et du salariat au Japon, afin de montrer l'importance du rôle social de l'entreprise dans la société japonaise, perceptible dans la part qu'elle a eue dans la construction du système de classification. Cette esquisse est structurée autour des formes d'emploi et de la mobilité professionnelle. Elle examine l'émergence et la stabilisation de la norme dite de «l'emploi à vie», et met en lumière comment ce processus $\mathrm{a}$, simulta-

(1) Cette approche s'inscrit plus généralement dans les travaux anthropologiques sur le système de classification ( $c f$. DougLas, 1986; Douglas, Hill, 1992). Pour la France on peut évoquer A. Desrosières $(1988,2000,2001,2003)$.

(2) De même, le système de production statistique au Japon n'est pas analysé. La question de la place de l'État dans ce système devrait être étudiée pour mieux préciser le rôle que jouent les modalités de l'observation statistique aussi bien dans la mise en place des catégories que dans les politiques publiques. 
nément, fait émerger d'autres catégories d'emploi. La seconde partie de l'article présente les grandes enquêtes japonaises sur l'emploi, conduites par le Statistics Bureau of Ministry of Internal Affairs and communications du Japon. Les catégories d'emploi qu'elles utilisent sont analysées dans la dernière partie de l'article sous l'angle du statut d'emploi et de la forme d'emploi, mettant en évidence un cumul de significations successives à travers l'histoire.

\section{Les entreprises japonaises et le salariat}

$\mathrm{Au}$ Japon, de nombreux éléments pris en charge en France par les pouvoirs publics ont été historiquement dévolus aux entreprises, selon des rapports sociaux structurant les catégories d'emploi en fonction des attributs d'âge ou de genre des individus.

\section{Jusqu'à la fin de la Seconde Guerre mondiale}

Dans la seconde moitié du XIX $\mathrm{X}^{\mathrm{e}}$ siècle, les entreprises d'État jouèrent un rôle essentiel dans l'industrie lourde à cause de l'importance de la production militaire. Cette industrie profita largement des deux guerres sino-japonaise (1894-1895) et russo-japonaise (1904-1905). Par la suite, son développement s'est accompagné d'une modernisation technologique réalisée surtout grâce à la coopération des entreprises occidentales au cours des années 1920.

Dans le secteur des industries légères, le relais de l'État fut pris par des entreprises privées dans les années 1880 et 1890 . Ainsi l'industrie textile s'attacha très tôt à combler son retard sur la technologie occidentale, et les progrès techniques y furent comparativement plus rapides.

Par ailleurs, le développement de l'industrie lourde impliquait une évolution dans la formation et la qualification ouvrière. Dans ce secteur, le système de formation le plus ancien était l'institution des oyakata (maître/père), dont l'origine se trouve dans le milieu artisanal traditionnel. La majeure partie des ouvriers était placée sous le contrôle des oyakata, intermédiaires entre les ouvriers et l'entrepriseemployeur. Il s'agissait d'ouvriers ayant acquis une compétence grâce à leurs contacts avec d'autres ouvriers qualifiés ou avec des ingénieurs étrangers. Certains oyakata contrôlaient des centaines de travailleurs et étaient, de fait, des sous-traitants de l'entreprise. L'entreprise confiait à un oyakata une tâche à accomplir et celui-ci organisait le travail et la rémunération comme il l'entendait (HAZAMA, 1976, 1978; Gordon, 1988; Thomann, 2005).

L'apprentissage était fondé sur l'observation et l'imitation: les apprentis se formaient en regardant faire l'oyakata et les ouvriers plus âgés. Cependant, pour répondre aux besoins de formation plus systé- matiques des ouvriers, le gouvernement mit en place des écoles techniques dès la fin du XIXe siècle, suivi par les grandes entreprises privées. Ce type de formation se répandit progressivement avant la Première Guerre mondiale (Hazama, 1978). Après la guerre, les grandes entreprises se mirent à pratiquer systématiquement la formation interne, qu'elles dispensaient aux jeunes recrutés directement après leur formation scolaire (SumIYA, 1977). Le remplacement d'une qualification artisanale et la disparition de l'institution des oyakata, de pair avec l'introduction de l'Organisation scientifique du travail, marquent pour la première fois l'apparition de relations salariales directes entre les travailleurs et l'entreprise dans le secteur de l'industrie lourde. Par contre, dans l'industrie légère, notamment textile, l'institution des oyakata n'existait pas. La mécanisation qui caractérisait cette industrie favorisait le contrôle direct de la main-d'œuvre par l'entreprise, main-d'œuvre composée essentiellement de jeunes femmes dont la formation se faisait presque exclusivement sur le tas. Il existait des écoles d'entreprise, mais ces établissements enseignaient les arts domestiques féminins.

Après la Première Guerre mondiale se met en place une population ouvrière qui constituera, ultérieurement, le cœur de la norme d'emploi dominante: celle des ouvriers qualifiés de l'industrie lourde, appartenant surtout à de grandes entreprises, ouvriers toute leur vie et sans autres ressources. Par ailleurs, la grande partie des ouvriers non qualifiés effectuait un travail saisonnier durant la saison creuse agricole, ou bien restait fermiers et travaillait temporairement dans les usines (3).

La disparition progressive du système des oyakata s'est accompagnée d'une politique d'entreprise visant à stabiliser les ouvriers qualifiés, très demandés, mais qui changeaient fréquemment d'emploi à la recherche de meilleures conditions de travail. Dès les années 1910, les grandes entreprises ont introduit des politiques d'avantages sociaux appliqués exclusivement aux ouvriers qualifiés, consolidant ainsi la distinction entre ouvriers réguliers qualifiés (jôko ou jôyô koyô) et ouvriers temporaires non ou peu qualifiés. Cette politique ne s'appliquait pas uniformément à tous les ouvriers permanents; elle touchait surtout les plus anciens. Tous ces éléments - politique de formation, établissement d'un statut privilégié, attribution d'avantages sociaux - aboutirent à la constitution d'un marché du travail fermé où la mobilité était essentiellement interne à l'entreprise. Le salaire

(3) Le travail «en alternance» entre l'industrie et la campagne, appelé dekasegi, caractérise une partie de la main-d'œuvre japonaise jusqu'à nos jours (voir ÔKAWA, 1979; WATANABE, HANEDA, 1977). Le terme dekasegi est actuellement employé pour désigner les immigrés issus de la migration japonaise outre-mer, principalement en Amérique du Sud, qui retournent au Japon pour y travailler. 
à l'ancienneté fait partie de cette stratégie de stabilisation de la main-d'œuvre qualifiée.

Les relations salariales qui se structurent ainsi dans la première moitié du $\mathrm{XX}^{\mathrm{e}}$ siècle doivent être replacées dans un contexte historique dans lequel les instances politiques et économiques sont profondément imbriquées avec la structure de parenté. L'État et l'entreprise étaient présentés comme un prolongement de l'organisation familiale. Dans le discours politique et patronal, le modèle de référence était la famille patriarcale dans laquelle la continuité du lignage était un absolu. La piété filiale acquise dans la famille, décrite comme le fondement de la morale et de l'éthique, était en continuité directe avec la loyauté envers l'empereur. L'effort des organisations politiques et économiques pour étendre ces règles familiales à l'organisation du travail est repérable dans les discours patronaux contre la promulgation $\mathrm{du}$ Code du travail, lequel risquait d'introduire, selon eux, un cadre juridique nouveau et externe dans les relations salariales, alors que ces dernières devraient être régies dans le cadre de la parenté.

Pendant la Seconde Guerre mondiale, et surtout vers sa fin, le salaire a progressivement été dissocié du travail effectué, et a acquis un caractère de revenu de survie pour le travailleur et sa famille, dans le cadre de la mobilisation pour la guerre. Ainsi, la famille d'un ouvrier mobilisé continuait à recevoir son salaire pendant son absence, ce salaire étant considéré comme une rétribution du soldat de l'armée impériale. Cette dissociation du salaire et du travail effectif, son association à la survie de la famille auraient préparé la forme du salaire dit «vital», instituée après guerre à la suite d'une forte mobilisation syndicale (MAGOTA, 1978).

\section{La fin de la guerre et la période de haute croissance économique}

Après la guerre, les syndicats mobilisent contre les licenciements, revendiquant la sécurité d'emploi et un salaire minimum basé sur le coût de survie. La partie centrale du salaire, qui a marqué le système ultérieur, était calculée en fonction de l'âge du travailleur, et avait comme objectif d'assurer la vie du salarié et de sa famille. À cette partie s'ajoutaient des éléments prenant en compte la compétence et l'ancienneté (Urabe, ÔMURA, 1983).

Cette forme du salaire est fortement liée à l'institution familiale. À travers la sécurité d'emploi et le revenu du chef de famille masculin, l'entreprise est censée assurer la survie de la famille. Dans ce cadre, la fonction de régulation de l'emploi ainsi que la protection sociale étaient déléguées en partie à l'entreprise. De même, l'institution familiale, intégrée dans cette configuration, fournissait la base de services aux membres de la famille à la place des organismes publics ou d'État : les femmes assuraient ces services, jouant le rôle combiné de travailleuse/ mère/épouse/bru, parfois en se retirant du marché du travail, et souvent en travaillant en tant qu'aide familiale ou à temps partiel.

Pendant la période de forte croissance économique, 1955-1970, les grandes entreprises japonaises évitaient autant que possible les compressions d'effectifs; elles ont ainsi progressivement institué la norme d'emploi dite «emploi à vie», shûshin koyô.

C'est à travers le développement du débat sur la gestion «à la japonaise», dans lequel les médias ont joué un rôle très important, que la pratique de l'emploi à long terme, apparue dans les années 1920 dans le contexte de la modernisation de l'industrie lourde, défendue par les syndicats d'entreprise après la Seconde Guerre mondiale, se voit instituée comme concept clé de la forme d'emploi (Nomura, 1994; Sugita, 1989). Les termes d' «emploi à vie» (shûshin koyô) et "salaire à l'ancienneté» (nenkô chingin) se stabilisent et entrent dans le langage ordinaire au cours des années 1960 et 1970.

\section{La restructuration de l'organisation de la production}

Vers la fin des années 1960 et au début des années 1970, les grandes entreprises se lancent à la fois dans la rationalisation intérieure et dans la réorganisation des sous-traitants. La grande majorité des entreprises japonaises est intégrée dans des chaînes de production chapeautées par une grande entreprise de plus en plus spécialisée dans la conception et la commercialisation. La production est assurée par une cascade de sous-traitants qui sont organisés de manière extrêmement hiérarchisée. Le modèle de référence pour une entreprise japonaise serait de démarrer au bas de l'échelle, puis de grimper en assumant des processus de production de plus en plus difficiles du point de vue technologique ou organisationnel, et en créant ses propres réseaux de sous-traitants. Cette ascension a des conséquences économiques immédiates, en particulier au niveau des prix et des salaires, car le prix de l'heure achetée/ vendue dans les transactions dépend de la place de l'entreprise dans cette hiérarchie. L'ascension de l'entreprise dans l'échelle a une répercussion directe sur le personnel. Du fait du statut plus élevé de son entreprise, le salarié jouira lui-même d'un prestige et d'un salaire plus élevés, et d'une meilleure sécurité de l'emploi. Ainsi, la mobilité est située plutôt au niveau de l'entreprise qu'au niveau de l'individu. La carrière professionnelle d'un individu est étroitement liée à l'histoire de l'entreprise. Par ailleurs, jusqu'à la crise actuelle, l'entreprise japonaise fonctionnait sur la base d'un champ d'action temporel très long. Les participations croisées dans les grandes entreprises, l'engagement à long terme de la banque principale, permettaient aux entreprises d'adopter une logique stratégique à un horizon temporel fort 
éloigné. Dans ce cadre, avec la norme d'emploi à vie, les membres d'une entreprise partageaient intimement un vécu et un avenir collectifs (MAGAUD, Sugita, 1993 ; Sugita, 1997).

\section{La politique de flexibilisation du marché de l'emploi et l'éclatement des bulles financières}

En 1985, une nouvelle loi, the Worker Dispatching Law(4), autorise les entreprises intérimaires dans certains secteurs d'activité; en 1999, un amendement à la loi les autorise dans presque tous les secteurs, sauf dans des cas bien précis. Un dernier amendement, en 2004, a prolongé la durée du contrat d'un an à trois ans sous certaines conditions.

L'éclatement des bulles financières en 1992 et la crise économique qui a suivi ont accéléré l'effritement du système d'emploi, suscitant la crise de la norme de «l'emploi à vie», et menaçant une population autrefois bien protégée, à savoir les salariés réguliers masculins âgés de 35 ans à 54 ans, chefs de famille, travaillant dans des grandes entreprises. L'emploi dit atypique augmente de manière forte depuis les années 1980 et en particulier dans les années 1990, et participe à la déstabilisation de la norme principale d'emploi. La hausse du taux de chômage, en particulier chez les jeunes, et l'instabilité croissante des trajectoires professionnelles modifient profondément les perspectives de vie. Le licenciement et la difficulté de retrouver un emploi chez les hommes ayant connu une longue carrière stable bouleversent la personne et menacent la division sexuelle du travail au sein de la famille (Kase, Sugita, 2006).

\section{Les enquêtes japonaises sur l'emploi}

Les catégories d'emploi au Japon vont être étudiées maintenant à partir des définitions qui en sont données dans les questionnaires des trois enquêtes conduites par le Statistics Bureau of Ministry of Internal Affaires and Communications du Japon. Ce sont les enquêtes de base sur l'emploi au Japon. Conçues et réalisées par le même organisme, elles sont relativement homogènes, ce qui va faciliter le travail de déconstruction des catégories utilisées.

Le questionnaire n'est pas rempli par l'enquêteur dans le cadre d'un entretien en face à face, mais distribué par l'enquêteur qui le récupère ensuite chez les répondants.

\section{Labour Force Survey}

L'équivalent de l'Enquête Emploi, rôdô ryoku chôsa - Labour Force Survey (LFS ci-après) - a été introduit au Japon en 1946 sous la direction des Américains suivant le modèle du Current Population Survey (CPS ci-après) aux États-Unis (IWAI, 1992). La LFS a commencé véritablement en 1947 après un an d'essai. Elle est réalisée auprès d'environ 40000 ménages, soit environ 100000 personnes de 15 ans et plus. Le même échantillon est suivi pendant deux mois. Elle porte sur la dernière semaine de chaque mois (dans le cas du mois de décembre, il s'agit de la semaine du 20 au 26). Cette enquête ainsi que la Special Survey of the LFS (voir ci-dessous), suit la convention du $\mathrm{BIT}$, et porte sur la semaine de référence.

La LFS comprend en sus des questions concernant l'identité, la position au sein du ménage et l'état civil: neuf questions au total pour les personnes actives occupées, deux questions pour les personnes qui n'ont pas travaillé dans la semaine de référence et qui cherchent un emploi, et une question pour les inactifs. La différence dans la taille du questionnaire entre la LFS d'une part et la CPS ou l'Enquête Emploi en France de l'autre est flagrante: la partie du questionnaire de la CPS sur l'emploi comporte environ 200 questions. Le questionnaire de l'Enquête Emploi comporte, pour les personnes ayant une activité professionnelle, grosso modo une soixantaine de questions (nombre variable selon les filtres), et une trentaine de questions pour les personnes s'étant déclarées «chômeur».

Cette enquête de taille restreinte est complétée par la Special Survey of the Labour Force Survey (SSLFS ci-après), conduite une fois par an à peu près sur un échantillon de même taille - éventuellement un peu plus faible. Cette enquête a été réalisée pour la première fois en 1949 en tant qu'enquête complémentaire à la LFS. Elle porte le nom de SSLFS à partir de 1962. Depuis 1999, elle est effectuée deux fois par an. À partir de 2002, elle est intégrée dans la LFS en tant que questionnaire spécial. J'examinerai ici le questionnaire SSLFS aussi bien que le questionnaire spécial de la LFS qui l'a remplacé. Quant au volume, le questionnaire de 1964 comporte, sur l'emploi, cinq questions au plus pour les actifs, et quatre questions pour les inactifs. Le nouveau questionnaire de 2002 comprend treize questions au plus pour les personnes de différents statuts (actif occupé, chômeur, inactif).

\section{Employment Status Survey (Shûgyô kôzô kihon chôsa)}

Cette enquête a démarré en 1956 au rythme d'une enquête tous les trois ans. À partir de 1982, elle s'est déroulée tous les cinq ans. Elle porte sur environ 430000 ménages, correspondant à peu près à 1100000 personnes de 15 ans et plus. À la 
différence des deux enquêtes précédentes, celle-ci n'adopte pas la convention du BIT, et est centrée sur l'état «habituel» de la situation professionnelle du répondant. Le questionnaire de 1956 comprend neuf questions au plus pour les personnes avec un travail, six questions pour les personnes sans travail et qui souhaitent travailler, et trois questions pour les personnes sans travail et qui ne désirent pas travailler. Le questionnaire de 2002 comporte, pour les personnes ayant une activité professionnelle, une trentaine de questions au plus, une vingtaine pour les personnes sans travail et cherchant un emploi, et une douzaine au plus pour les inactifs.

\section{Les catégories d'emploi dans les enquêtes}

La petite taille des questionnaires que nous venons de constater est certainement liée à la méthode de collecte. Toutefois, il ne suffit pas de réduire la taille du questionnaire pour rendre cette méthode opérationnelle. Il existe certes un manuel d'aide pour remplir le questionnaire, mais il ne peut pas remplacer l'enquêteur, censé préparer le répondant à l'enquête (5), l'aider à comprendre le sens des questions, et tenter d'éviter de possibles erreurs d'interprétation, etc. Plus fondamentalement, cette méthode implique que les catégories proposées aux répondants soient assez nombreuses et qu'il $\mathrm{y}$ ait une bonne adéquation des catégories statistiques avec les catégories du sens commun. Est-ce le cas? Si oui, comment cela fonctionne-t-il? Les catégories statistiques qui sont, par nécessité, rigoureuses et stabilisées (du moins est-ce leur vocation), peuvent-elles être suffisamment en adéquation avec les catégories de la vie quotidienne, et ceci sur une durée suffisamment longue, pour permettre l'analyse de l'évolution de l'emploi sur le long terme?

Partant ces questionnements, je vais à présent examiner les catégories d'emploi telles qu'elles apparaissent dans les questionnaires selon le système de classification adopté par les enquêtes.

Les catégories d'emploi proposées par les trois enquêtes(6) concernent le «statut d'emploi» (jyûgyô jô no chii) et la "forme d'emploi» (koyô keitai). Ces deux classifications sont fondées sur la déclaration du répondant, qui choisit parmi une liste de réponses.

\section{Le statut d'emploi}

Les éléments suivants figurent au début du questionnaire de la LFS.

Tableau 1 - Questionnaire de la LFS

Shigoto no naiyô : description détaillée du travail

- Remplissez la partie qui concerne le travail effectué durant la dernière semaine du mois (dans le cas du mois de décembre pour la période qui s'étend entre le 20 et le 26) ou le travail dont vous étiez temporairement absent durant la même semaine.

- Si vous avez effectué plus d'un travail, remplissez la partie qui concerne le travail pour lequel vous avez passé le plus d'heures.

\begin{tabular}{|c|c|c|}
\hline \multirow{6}{*}{$\begin{array}{l}\text { Jyûgyô jô no chii (statut d'emploi) } \\
\text { - Rinji yatoi no hito (temporaire) s'applique } \\
\text { aux personnes dont la durée du contrat est } \\
\text { d'un mois et plus, et inférieure à un an } \\
\text { - Hiyatoi no hito (journalier) s'applique aux } \\
\text { personnes dont la durée du contrat est infé- } \\
\text { rieure à un mois } \\
\text { - Jiei gyôshu (indépendant) signifie le proprié- } \\
\text { taire de magasin, l'exploitant agricole, etc. } \\
\text { à son propre compte } \\
\text { - Naishoku (travail domestique) signifie } \\
\text { le travail à la pièce exécuté à la maison }\end{array}$} & \multirow[t]{2}{*}{ Yatowarete iru hito no uchi : salarié } & $\begin{array}{l}\text { 1) Jôko no hito : salarié régulier } \\
\text { 2) Rinji yatoi no hito: temporaire } \\
\text { 3) Hiyatoi no hito : journalier }\end{array}$ \\
\hline & & $\begin{array}{l}\text { 4) Kaisha nado no yakuin: directeur } \\
\text { des firmes, etc. }\end{array}$ \\
\hline & \multirow{2}{*}{ Jiei gyôshu : indépendant } & 5) Yatoi nin ari : avec salariés \\
\hline & & 6) Yatoi nin nashi: sans salariés \\
\hline & & $\begin{array}{l}\text { 7) Jika eigyô no tetsudai : aide } \\
\text { de l'entreprise familiale indépendante }\end{array}$ \\
\hline & & 8) Naishoku : travail domestique \\
\hline
\end{tabular}

(5) L'enquêteur-distributeur de questionnaires présente l'enquête au répondant lors de son passage, mais il ne peut pas faire un travail relationnel de même qualité que l'enquêteur qui reste pour l'entretien. Par contre, la présence de celui-ci introduit le difficile problème des relations entre l'enquêteur et l'enquêté qui influenceraient les réponses.

(6) Voir glossaire en annexe. 
Comme nous pouvons le constater dans la colonne de gauche, le questionnaire ne donne aucune explication sur jôko(7) no hito, salarié régulier(8). La définition se construit par élimination, à partir des définitions des autres catégories de salariés. Ce caractère potentiellement non-substantiel de la catégorie d'emploi régulier (jôko) trouve son écho dans les modifications de sa définition. En effet, dans la terminologie utilisée dans les rapports de l'enquête LFS (terminologie inconnue des répondants), la définition de jôko se modifie au fil des années. De 1964 à 1970, jôkô est défini comme suit: «Personnes employées soit sans que soit fixée la durée d'embauche, soit pour une durée déterminée d'un an et plus, ainsi que directeurs des firmes, des associations et des organisations diverses». Entre 1971 et 1987, jôkô est défini comme "personnes autres que les rinji yatoi (temporaires) et hiyatoi (journaliers)». Entre 1988 et 1990, ce terme définit «les personnes employées sans détermination de durée ou pour une durée déterminée d'un an et plus». Entre 1991 et 1996 , cela devient «parmi les salariés, ceux qui sont autres que rinji yatoi et hiyatoi ». À partir de 1997, la définition est la suivante:

Tableau 2 - Définition de la catégorie Jôko à partir de 1997

\begin{tabular}{|l|l|l|}
\hline \multicolumn{2}{|l|}{ Salariés réguliers (jôko) dont } & \\
\hline Directeurs (yakuin) & $\begin{array}{l}\text { Directeurs des firmes, associations et organisations } \\
\text { diverses }\end{array}$ \\
\cline { 2 - 4 } & $\begin{array}{l}\text { Salariés réguliers ordi- } \\
\text { naires (ippan jôko) }\end{array}$ & $\begin{array}{l}\text { Personnes employées pour une durée d'un an et plus, } \\
\text { ou qui travaillent sur un contrat à durée indéterminée } \\
\text { (sauf les directeurs) }\end{array}$ \\
\hline $\begin{array}{l}\text { Salariés temporaires (rinji } \\
\text { yatoi) }\end{array}$ & $\begin{array}{l}\text { Personnes employées pour une durée déterminée d'un } \\
\text { mois et plus, et inférieure à un an }\end{array}$ \\
\hline Salariés journaliers (hiyatoi) & $\begin{array}{l}\text { Personnes employées sur une base journalière ou pour } \\
\text { une durée déterminée inférieure à un mois }\end{array}$ \\
\hline
\end{tabular}

Ce qui nous intéresse ici est le fait que, même dans la terminologie, la définition de jôko ne soit pas constante, et qu'à certaines périodes, ce soit une définition par défaut, à travers les définitions retenues pour les autres catégories d'emploi. Le fait que la catégorie d'emploi censée être au cœur du système soit définie via les autres catégories d'emploi mérite réflexion. Quant au questionnaire, l'enquêté doit procéder à une interprétation afin de savoir ce que c'est le jôko no hito, d'autant plus que jôko n'est pas un terme courant. Ce mot est l'abréviation de jôyô koyô, qui signifie emploi permanent, ce qui n'est absolument pas évident pour les personnes enquêtées. Ainsi que nous l'avons vu, ce terme était historiquement employé pour désigner les travailleurs permanents et les différencier des autres

(7) Peut être lu comme jôyatoi également.

(8) Traduit en anglais comme "regular employee» dans le rapport de l'enquête. $C f$. plus loin. travailleurs. De nos jours, il n'est pas utilisé dans la vie quotidienne. Par contre, rinji yatoi (temporaire) et hiyatoi (journalier) sont parfaitement compréhensibles (9).

Ainsi que nous le voyons dans la terminologie présentée ci-dessus, le Statistics Bureau donne comme définition du «salarié régulier ordinaire» des personnes employées pour une durée d'un an et plus, ou qui travaillent sur un contrat à durée indéterminée. Toutefois, dans la pratique, la plupart des «salariés réguliers ordinaires» n'ayant pas de contrat de travail, c'est plutôt l'opération par élimination qui prévaut.

Cenpendant, un problème se pose lorsque le questionnement est «qui sont ceux qui n'ont pas de contrat de travail dans l'entreprise?». C'est-àdire qui sont les personnes qui, dans la pratique sociale, entrent dans la catégorie de jôko, selon la définition donnée dans l'enquête? En effet, la plupart des travailleurs à temps partiel, les jeunes recrutés temporairement, c'est-à-dire les personnes qui seraient classées comme occupant les formes d'emploi dites atypiques, n'ont pas de contrat. Ainsi, de nos jours, la catégorie jôko, héritée de l'époque où jôkô koyô, l'emploi permanent, émergea en opposition à la catégorisation des travailleurs à mobilité très élevée ou des jeunes ouvrières dans le secteur de l'industrie légère, peut comprendre en son sein des formes d'emploi atypiques. Effectivement, l'Employment Status Survey (ESS ci-après) fournit un tableau croisé de la classification par statut et par forme d'emploi dans lequel figure le temps partiel, arubaito, le travail intérimaire, et le travail sous contrat dans la catégorie jôko comme dans les autres catégories. La SSLFS ainsi que l'ESS procèdent de la même manière que la LFS. Ni l'une ni l'autre ne fournissent la définition du «salarié régulier» (jôko) dans le questionnaire. Seule la SSLFS donne une définition dans le manuel d'aide pour remplir le questionnaire: «Jôko désigne la personne employée avec un contrat d'un an et plus ou avec un contrat à durée indéterminée».

Le caractère non rigoureux et potentiellement équivoque de la catégorie de jôko se retrouve également dans la traduction anglaise de ce terme utilisée dans les rapports d'enquêtes. erronée par rapport à la définition donnée par l'enquête. 
Traduction anglaise de jôko

\begin{tabular}{|c|c|c|}
\hline LFS & SSLFS & ESS \\
\hline Regular employee & Regular employee & Ordinary employee \\
\hline
\end{tabular}

Le même mot anglais, "Regular employee» apparaît dans la classification par la «forme d'emploi» (koyô keitai) (voir ci-dessous).

Traduction anglaise de seiki no shokuin, jyûgyôin

\begin{tabular}{|c|c|c|}
\hline LFS & SSLFS & ESS \\
\hline & Regular staff & Regular employee \\
\hline
\end{tabular}

Or, il s'agit de la traduction des termes seiki no shokuin, et jyûgyôin, "salarié typique», excluant donc les formes d'emploi atypiques (10). Comment le même terme peut-il désigner à la fois une catégorie incluant des formes d'emploi atypiques et une autre qui les exclut? Cette ambiguïté ou plutôt cette contradiction, est d'autant plus dérangeante que ces trois enquêtes partagent par ailleurs de nombreux concepts. Elle semble être révélatrice d'une accumulation, d'une stratification de significations successives à travers l'histoire. Afin d'examiner cette hypothèse, nous allons passer à présent au second système de classification.

\section{La forme d'emploi}

Dans le questionnaire de la SSLFS ainsi que dans le nouveau questionnaire spécial de la LSF comme dans l'ESS, figure une question aux répondants se déclarant comme salariés (à l'exclusion des directeurs), quant à leur appellation sur le lieu de travail. Les différentes appellations parmi lesquelles on leur demande de choisir sont identiques pour les deux derniers questionnaires.

Le questionnaire SSLFS ne donne pas de définition de différentes «formes d'emploi», ni dans le questionnaire lui-même, ni dans le manuel d'aide. En revanche, le questionnaire spécial de la LFS, qui a remplacé la SSLFS depuis 2002, fournit les définitions suivantes dans le manuel d'aide:
On peut remarquer que, tandis que les autres termes ne sont pas accompagnés de précision, seul haken shain est explicité déjà dans le questionnaire. Ce statut particulier de haken shain se retrouve dans le cas de l'ESS, qui fournit l'explication suivante:

\begin{tabular}{|l|}
\hline Question A1-2 : appellation sur le lieu de travail \\
\hline - Le haken shain, envoyé par l'agence de travail intéri- \\
maire » désigne les personnes encadrées par le Worker \\
Dispatching Law. \\
- Pour les salariés haken dans les magasins de grande \\
surface, ou les gardiens haken, référez-vous à l'appellation \\
qui était employée à l'agence intérimaire qui vous a envoyé.
\end{tabular}

De même, dans le manuel d'aide, l'ESS donne l'explication suivante:

- Si vous n'êtes pas sûr que vous êtes pâto ou arubaito, référez-vous à l'appellation qui a été utilisée dans l'annonce d'embauche ou dans les textes d'embauche ou encore lors du contrat d'embauche.

- Pour les personnes envoyées par l'agence de travail intérimaire, choisissez haken shain (intérimaire), même si d'autres appellations sont utilisées sur le lieu de travail.

L'explication concernant haken shain continue encore longuement dans le manuel ( $c f$. glossaire). Le fait que seule la catégorie haken shain fasse l'objet d'un long commentaire qui se réfère à la définition juridique suggère son caractère spécifique par rapport aux autres catégories d'emploi. D'où provient-t-il? Ces différentes formes d'emploi n'ont pas la même filiation historique. L'intérimaire et le contractuel sont plus récents; le premier est apparu dans le questionnaire en 1987, et le second en 2002, c'est-à-dire lors de la dernière enquête ESS. Les trois autres catégories ainsi que celle de «salarié ordinaire» sont des catégories plus anciennes. Le mot pâto taimu apparait pour la première fois dans le questionnaire ESS en 1968 comme une réponse - «Je souhaite être embauché à temps réduit (par exemple pâto taimu)» - à la question «Quelle sorte de travail souhaitez-

\begin{tabular}{ll}
\hline \multicolumn{2}{l}{ Question A4 : concernant votre travail actuel, si vous êtes salarié, indiquez l'appellation utilisée sur le lieu du travail } \\
\hline $\begin{array}{l}\text { Questionnaire } \\
\begin{array}{l}\text { Seiki no shokuin, } \\
\text { jyûgyôin }\end{array}\end{array}$ & Explication donnée dans le manuel d'aide \\
\hline $\begin{array}{l}\text { Pâto }{ }^{1} \text {, arubaito } 2 \\
\text { S'applique aux personnes appelées ippan shokuin (salarié ordinaire) ou seishain (salarié titulaire), etc. }\end{array}$ \\
$\begin{array}{l}\text { Haken shain (envoyé } \\
\text { par l'agence de travail } \\
\text { intérimaire) }\end{array}$ & $\begin{array}{l}\text { S'appliquent aux personnes qui sont appelées ainsi ou d'une manière similaire sur le lieu de travail sans } \\
\text { Dispatching Law }{ }^{3} \text {. Si votre cas correspond à cette catégorie, choisissez celle-ci, même si vous êtes appelé } \\
\text { par un autre terme listé ici }\end{array}$ \\
\hline Keiyaku shain & $\begin{array}{l}\text { S'applique aux personnes qui sont engagées avec un contrat à durée déterminée pour occuper un poste à } \\
\text { une compétence spécifique }\end{array}$ \\
\hline $\begin{array}{l}\text { Shokutaku } 4 \\
\text { S'applique aux personnes appelées ainsi ou de manière similaire sur leur lieu de travail, sans tenir compte } \\
\text { des conditions de travail ou de la durée du contrat }\end{array}$ \\
\hline
\end{tabular}

1 Le terme pâto taimu et son abréviation pâto sont des néologismes à partir de l'anglais «part time » (temps partiel).

2 Mot dérivé de l'allemand « arbeit », - travail.

3 Rôdôsha hakenhô.

4 Les personnes à qui on « confie » (shokutaku, traduit comme « entrust » en anglais) le travail. 
vous»? Dans la liste de réponses, on voit figurer, à côté de pâto, la réponse suivante: «Je souhaite être embauché avec un horaire normal - futsû kinmu).

La question concernant l'appellation sur le lieu de travail figurant dans le second système de classification est apparue en 1982. Les quatre réponses proposées sont: seiki no shokuin/jyûgyôin, pâto/arubaito, shokutaku etc., autres. Elles ont été introduites avant la mise en œuvre de la politique de flexibilisation du marché de l'emploi. L'intégration de l'intérimaire en 1987 et du contractuel en 2002 marque l'entrée d'un langage juridique dans ce système de classification qui était jusqu'alors fondé uniquement sur la dénomination ordinaire, plus précisément sur la pratique langagière de l'entreprise. Ainsi, dans le même système de classification, différentes couches de langage de codification peuvent s'accumuler. Les explications dans le manuel d'aide montrent bien que la cohabitation entre ces deux langages n'est pas encore stabilisée, et que le sens à donner aux mots n'est pas partagé de manière homogène par les salariés. L'histoire de la SSLFS est à peu près parallèle: le terme pâto taimu apparaît en 1967; en 1981, parmi les réponses figurent pour la première fois seiki jyûgyôin ainsi que arubaito, marquant la formalisation concomitante de ces catégories. Quant à la question concernant l' «appellation sur le lieu de travail», elle entre dans le questionnaire SSLFS en 1983 lorsque figure pour la première fois shokutaku. L'intérimaire apparaît en 1999, et le contractuel a été intégré en 2002.

Nous avons vu que la désignation de la plupart des formes d'emploi n'est pas fondée sur le contrat de travail, car, dans la grande majorité des cas, il n'existe pas de contrat légal. Normalement il devrait exister un règlement intérieur d'entreprise définissant les statuts et les formes d'emploi, mais souvent il n'y en a pas ou s'il y en a un, ce règlement est souvent caduc. De manière générale, l'emploi au Japon est relativement peu régulé par un cadre juridique. En lieu et place d'un système légal relevant des pouvoirs publics et dépassant ainsi le niveau de l'entreprise, il revient à cette dernière d'encadrer le statut de l'emploi(11). Cela ne signifie pas que l'entreprise soit libre de disposer de ses salariés comme elle l'entend. Cette pratique d'emploi fondée sur un consensus social concernant le rôle de l'entreprise dans la société est effectivement sanctionnée par la société et protégée par la jurisprudence(12) (Sugeno, 2004).

(11) Le cadre législatif existe, bien entendu, mais d'un côté, il laisse beaucoup plus d'espace à l'entreprise comparativement à ce qui se passe en France, et de l'autre côté, il est amendé par une nombre important de dérogations.

(12) C'est bien pour cette raison que la vague de licenciements massifs dans les grandes entreprises a suscité une angoisse au sein de la société japonaise. Non seulement du fait de l'importance numérique de ces licenciements, mais plus encore parce que le consensus social ne tient plus. Il s'agit bien de l'effritement d'une norme.
Si c' est une logique juridique qui donne de la cohérence aux deux formes les plus récentes d'emploi, quelle est la logique qui sous-tend les quatre autres formes? Ces formes d'emploi plus anciennes, «titulaire», «temps partiel», «arubaito», «shokutaku», pour lesquels les questionnaires n'expliquent pratiquement rien, ce qui laisse supposer une multiplicité de sens, sont très fortement liées au genre et au cycle de vie. Le temps partiel concerne les femmes de plus de 35 ans, c'est-à-dire celles qui reviennent sur le marché de l'emploi après avoir élevé leurs enfants. L'arubaito est le fait des jeunes, garçons et filles. En fait, il s'agit de «petits boulots». Les pâto aussi bien que les arubaito sont payés à l'heure, et sont exclus des avantages sociaux accordés par l'entreprise. Le shokutaku est lié aux hommes âgés de plus de 55 ans, et surtout de plus de 65 ans. Dans la pratique, il s'agit d'hommes qui continuent de travailler au sein de l'entreprise après avoir pris leur retraite, en tant que salariés titulaires. Enfin la notion de «titulaire» est fortement liée aux hommes adultes ( $c f$. tableau 3 ).

Ainsi, c'est la construction sociale des formes d'emploi, intégrant les relations de genre, d'âge, le rôle de l'institution familiale et la division sexuelle du travail, qui sous-tend la cohérence de ce système de classification. Cette construction sociale fournit la base de l'adéquation entre les catégories ordinaires et les catégories statistiques, et ainsi étaie la validité opérationnelle lors de la collecte d'information.

\section{Changements historiques et tensions entre catégories d'emploi}

Au-delà de l'opérationnalité des catégories d'emploi, le fait que ce système de classification savant se base sur les pratiques de dénomination au sein de l'entreprise donne une bonne indication sur la place de l'entreprise dans la société japonaise, à côté de l'État et d'autres institutions publiques : bien des éléments pris en charge par les pouvoirs publics en France sont, au Japon, dévolus à l'entreprise avec l'accord des partenaires sociaux. Le consensus social s'est longtemps appuyé sur le fait que l'entreprise assurait la stabilité à long terme des revenus des ménages à travers le salaire du chef de famille travaillant en son sein. Ce consensus structure les catégories d'emploi en les mettant en relation avec les différents attributs sociologiques de l'individu, aussi bien du point de vue du genre que de la place du salarié dans le cycle de vie. Les formes d'emploi, «typique» et «atypique» par exemple, et leurs appellations expriment aussi bien la position de l'individu que la place de l'entreprise dans cette configuration.

Les deux langages, dans le second système de classification fondé sur la forme d'emploi, reflètent les tensions entre les deux champs, celui de la loi et celui de l'entreprise. Ces tensions semblent 
Tableau 3

Répartition des emplois selon leur forme

en 2001 (en \%)

\begin{tabular}{|c|c|c|c|c|c|c|c|c|}
\hline & & $\begin{array}{l}\text { Tous } \\
\text { âges }\end{array}$ & $\begin{array}{c}15-24 \\
\text { ans }\end{array}$ & $\begin{array}{c}25-34 \\
\text { ans }\end{array}$ & $\begin{array}{c}35-44 \\
\text { ans }\end{array}$ & $\begin{array}{c}45-54 \\
\text { ans }\end{array}$ & $\begin{array}{c}55-64 \\
\text { ans }\end{array}$ & $\begin{array}{l}65 \text { ans } \\
\text { et plus }\end{array}$ \\
\hline \multirow{9}{*}{$\begin{array}{l}\stackrel{\mathscr{U}}{\Xi} \\
\stackrel{\Xi}{\Xi} \\
\stackrel{\Xi}{\Xi}\end{array}$} & Directeurs & 9 & 0 & 2 & 7 & 11 & 19 & 32 \\
\hline & Seiki no shokuin, jyûgyôin & 78 & 64 & 90 & 89 & 83 & 62 & 26 \\
\hline & Emploi atypique & 13 & 35 & 8 & 4 & 6 & 19 & 42 \\
\hline & Pâto & 2 & 2 & 1 & 0 & 1 & 3 & 12 \\
\hline & Arubaito & 5 & 29 & 3 & 1 & 1 & 3 & 8 \\
\hline & Intérimaire & 0 & 1 & 0 & 0 & 0 & 0 & 1 \\
\hline & Contractuel et shokutaku & 4 & 3 & 2 & 2 & 2 & 10 & 16 \\
\hline & Autres & 1 & 1 & 1 & 1 & 1 & 3 & 5 \\
\hline & Total emploi & 100 & 100 & 100 & 100 & 100 & 100 & 100 \\
\hline \multirow{9}{*}{ 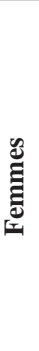 } & Directeurs & 4 & 0 & 1 & 4 & 6 & 8 & 32 \\
\hline & Seiki no yakuin, jyûgyôin & 51 & 57 & 66 & 47 & 43 & 39 & 25 \\
\hline & Emploi atypique & 45 & 43 & 33 & 49 & 51 & 53 & 41 \\
\hline & Pâto & 30 & 8 & 18 & 37 & 42 & 43 & 29 \\
\hline & Arubaito & 7 & 27 & 6 & 4 & 2 & 3 & 3 \\
\hline & Intérimaire & 2 & 1 & 3 & 2 & 1 & 0 & 0 \\
\hline & Contractuel et shokutaku & 5 & 5 & 4 & 4 & 4 & 5 & 5 \\
\hline & Autres & 2 & 1 & 2 & 2 & 1 & 2 & 5 \\
\hline & Total emploi & 100 & 100 & 100 & 100 & 100 & 100 & 100 \\
\hline
\end{tabular}

Sources: Special Survey of the Labour Force Survey.

augmenter dans la situation actuelle de crise où l'Etat tente de revenir sur le devant de la scène afin de prendre en charge les fonctions délaissées par les entreprises défaillantes.

Je voudrais revenir à présent sur le caractère, que j'ai qualifié de non-substantiel, du statut du salarié régulier (jôko) et sur le premier système de classification, basé sur le statut d'emploi. De même que l'ambiguïté et la tension dans le second système de classification relèvent du contexte historique, dans le premier système, le contexte historique met bien en évidence le caractère équivoque du statut du salarié régulier. En effet, lors de démarrage de ces enquêtes, les formes d'emploi qualifiées d'atypiques aujourd'hui, notamment le pâto (temps partiel), l'arubaito et le shokutaku n'étaient pas encore développées. Cela ne signifie pas que la population associée à ces formes d'emploi n'était pas présente sur le marché du travail. Prenons le cas des femmes. L'évolution du taux d'activité au Japon montre que les femmes japonaises maintiennent un taux d'activité assez stable ( $c f$. figure 1). Le changement s'observe plutôt dans la progression de la salarisation, en parallèle avec la forte diminution de l'aide familiale, en particulier dans le secteur de l'agriculture et de la forêt ( $c f$. tableau 4). Le travail à temps partiel apparaît vers le milieu des années 1950, son développement remonte aux années
1970, mais surtout après les années 1980. Lors des premières périodes des enquêtes sur la force du travail examinées ici, le brouillage de la catégorie jôko du fait de l'intégration des autres catégories d'emploi émergentes ne s'est pas encore produit. Au début, dans les années 1950 et sans doute dans la grande partie des années 1960, les trois statuts de salariés, jôko, temporaire et journalier étaient probablement aptes à appréhender le salariat.

Figure 1

Évolution du taux d'activité au Japon entre 1955 et 2005

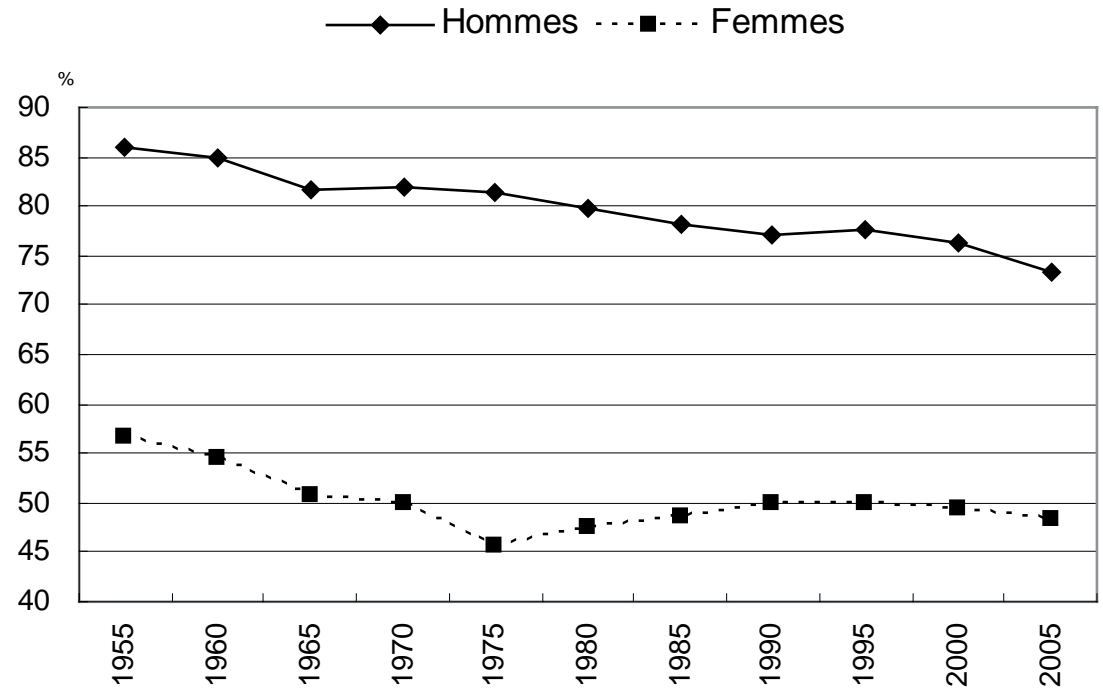

Sources: Labour Force Survey.

Champs : 15 ans et plus. 
Tableau 4

Évolution de la salarisation par sexe au Japon entre 1955 et 2005.

\begin{tabular}{|c|c|c|c|c|c|c|}
\hline & \multicolumn{3}{|c|}{ Hommes } & \multicolumn{3}{|c|}{ Femmes } \\
\hline & 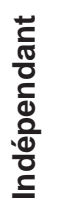 & 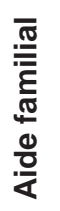 & $\begin{array}{l}\frac{0}{\frac{1}{2}} \\
\frac{\pi}{\sqrt[T]{D}}\end{array}$ & 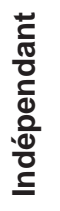 & 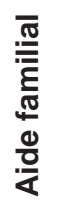 & $\frac{0}{\text { ஸे }}$ \\
\hline 1955 & 32 & 16 & 52 & 16 & 53 & 31 \\
\hline 1960 & 27 & 11 & 62 & 16 & 43 & 41 \\
\hline 1965 & 23 & 8 & 69 & 15 & 37 & 49 \\
\hline 1970 & 22 & 6 & 71 & 14 & 31 & 55 \\
\hline 1975 & 20 & 4 & 76 & 14 & 26 & 60 \\
\hline 1980 & 19 & 3 & 77 & 14 & 23 & 63 \\
\hline 1985 & 18 & 3 & 79 & 13 & 20 & 67 \\
\hline 1990 & 16 & 3 & 81 & 11 & 17 & 72 \\
\hline 1995 & 14 & 2 & 84 & 9 & 13 & 78 \\
\hline 2000 & 14 & 2 & 84 & 8 & 11 & 81 \\
\hline 2005 & 13 & 2 & 85 & 6 & 9 & 85 \\
\hline
\end{tabular}

Sources: Labour Force Survey.

Note:En 2005, 85\% des hommes occupés actifs sont salariés, $13 \%$ sont indépendants et $2 \%$ aides familiaux.

Champ: actifs occupés de 15 ans et plus.
On a remédié à l'effritement de la catégorie jôko et à l'insuffisance du premier système de classification fondé sur le statut d'emploi par l'introduction, en 1983, du second système de classification fondé sur la forme d'emploi et prenant mieux en compte la salarisation croissante et la diversification des formes d'emploi. Les catégories du premier système correspondaient à des périodes pendants lesquelles la part des indépendants et des aides familiaux demeurait importante. Dans le second système, les catégories sont issues des pratiques empiriques dont la sédimentation, appuyée par la jurisprudence, a assuré, du moins pour une certaine période, la stabilisation des catégories d'emploi.

Ainsi les catégories d'emploi sont sujettes aux changements, et les épaisseurs accumulées au fil du temps peuvent les rendre équivoques.

Il est évident que des comparaisons internationales appellent la mise en évidence préalable des contextes nationaux historiques et institutionnels dans lesquels les catégories ont émergé et ont évolué. La même précaution est nécessaire au niveau national, car les mêmes catégories d'emploi peuvent recouvrir des réalités différentes selon l'époque, le secteur d'activité, ou encore tout simplement selon l'enquête. Cette vigilance est d'autant plus indispensable que les épaisseurs de signification demeurent souvent implicites et inconscientes. 


\section{Bibliographie}

Desrosières A. (2000), «L'histoire de la statistique comme genre: styles d'écriture et usages sociaux», Genèses, 39.

Desrosières A. (2001), «Entre réalisme métrologique et conventions d'équivalence: les ambiguïtés de la sociologie quantitative», Genèses, 43.

Desrosières A. (2003), «Comment fabriquer un espace de commune mesure? Harmonisation des statistiques et réalisme de leurs usages », in: M. Lallement et J. Spurk (éds), Stratégies de la comparaison internationale, Paris, CNRS éditions.

Desrosières A., Thevenot L. (1988), Les catégories socioprofessionnelles, Paris, La Découverte.

Douglas M. (1986), How institutions think, New York, Syracuse University Press, (traduction française) Ainsi pensent les institutions, éditions Usher, 1989 et Comment pensent les institutions, Paris, La Découverte. 2004.

Douglas M., Hill D. éd. (1992), How classification works, Edinburgh, Edinburgh University Press.

Fus H. (1995), Nihongata kigyô shakai to josei rôdô. Shokugyô to katei no ryôritsu o mezashite, Kyoto, Minerva shobô.

Gordon A. (1988), The evolution of labour relations in Japan. Heavy Industry, 1853-1955, Council of East Asian Studies, Harvard University, Cambridge \& London.

Hazama H. (1976), "Formation of An Industrial Work Force: Historical Changes in the Life Style of Industrial Workers", in: Hugh Patrick (ed.), Japanese Industrialization and Its Social Consequences, Berkeley, University of California Press.

Hazama H. (1978), Nihon rômu kanri kenkyû, Ochanomizu shobô, Tokyo.

Hirata H., Sugita K. (1988), «Politique paternaliste et division sexuelle du travail: le cas de l'industrie japonaise», Le Mouvement Social, n 144.

IwaI H. (1992), Rôdôryoku, koyô, shitsugyô tôkei no kokusaiteki tenkai, Chiba, Azusa shuppan.

Kase K., Sugita K. éd. (2006), The Unemployed and Unemployment in an International Perspective: Comparative Studies of Japan, France and Brazil, ISS Research Series, Institut des Sciences Sociales, Université de Tokyo, Tokyo.
Magaud J., Sugita K. (1993), «Le retour des réseaux : à propos d'une comparaison franco-japonaise», Gérer et Comprendre, $\mathrm{n}^{\circ} 31$.

Magota R. (1978), Nenkô chingin no shûen, Nikkei shinsho, Tokyo.

Nomura M. (1994), Shûshin koyô, Iwanami, Tokyo.

Ôкаwа T. (1979), Dekasegi no keizaigaku, Kinokuniya, Tokyo.

SAкамото F. (1977), Nihon koyôshi volume 2. Nenkôsei heno nagai michinori, Chûôkeizaisha, Tokyo.

Sugeno K. (2004), Shin koyô shakai no hô, Yûhikaku, Tokyo.

Sugita K. (1989), «Le Japon: jeux de miroirs », Sociologie du travail, $\mathrm{n}^{\circ} 2$.

Sugita K (1997), «Sous-traitance en cascade au Japon: le cas d'un groupe d'entreprises dans le secteur électronique », Précarisation sociale, travail et santé, IRESCO/ CNRS.

Sumiya M, Kobayashi K., Hyodo Ts. (1967), Nihon shihonshugi to rôdô mondai, Tokyo daigaku shuppankai, Tokyo.

SumiYa M. (1976), Nihon chinrôdô no shiteki kenkyû, Ochanomizu shobô, Tokyo.

SumiYa M. éd. (1977), Nihon shokugyô kunren hattenshi, 2 vol., Nihon rôdô kyôkai, Tokyo.

Thomann B. (2005), «Japon. Les emplois non réguliers et les mécanismes de la régulation sociale», Chronique internationale de l'IRES, $\mathrm{n}^{\circ} 97$.

Thomann B. (2005), «Les conditions historiques de la naissance et de la reproduction de l'emploi à vie comme archétype de l'emploi masculin au Japon », Le Mouvement Social, $\mathrm{n}^{\circ} 210$.

Urabe K., Ômura K. (1983), Nihon teki rôshi kankei no tankyû, Chûô keizaisha, Tokyo.

Watanabe S., Haneda A. (1977), Dekasegi rôdô to nôson no seikatsu, Tokyo daigaku shuppankai, Tokyo.

Watanabe S. (1987), Dekasegi no sôgôteki kenkŷ̂, Tokyo daigaku shuppankai, Tokyo. 


\section{Glossaire}

La traduction anglaise provient de rapport annuel ou du site internet du Statistic Bureau (les mots clés sont traduits en français par l'auteure). En l'absence d'une traduction officielle en anglais, une traduction française est fournie à partir du texte japonais.

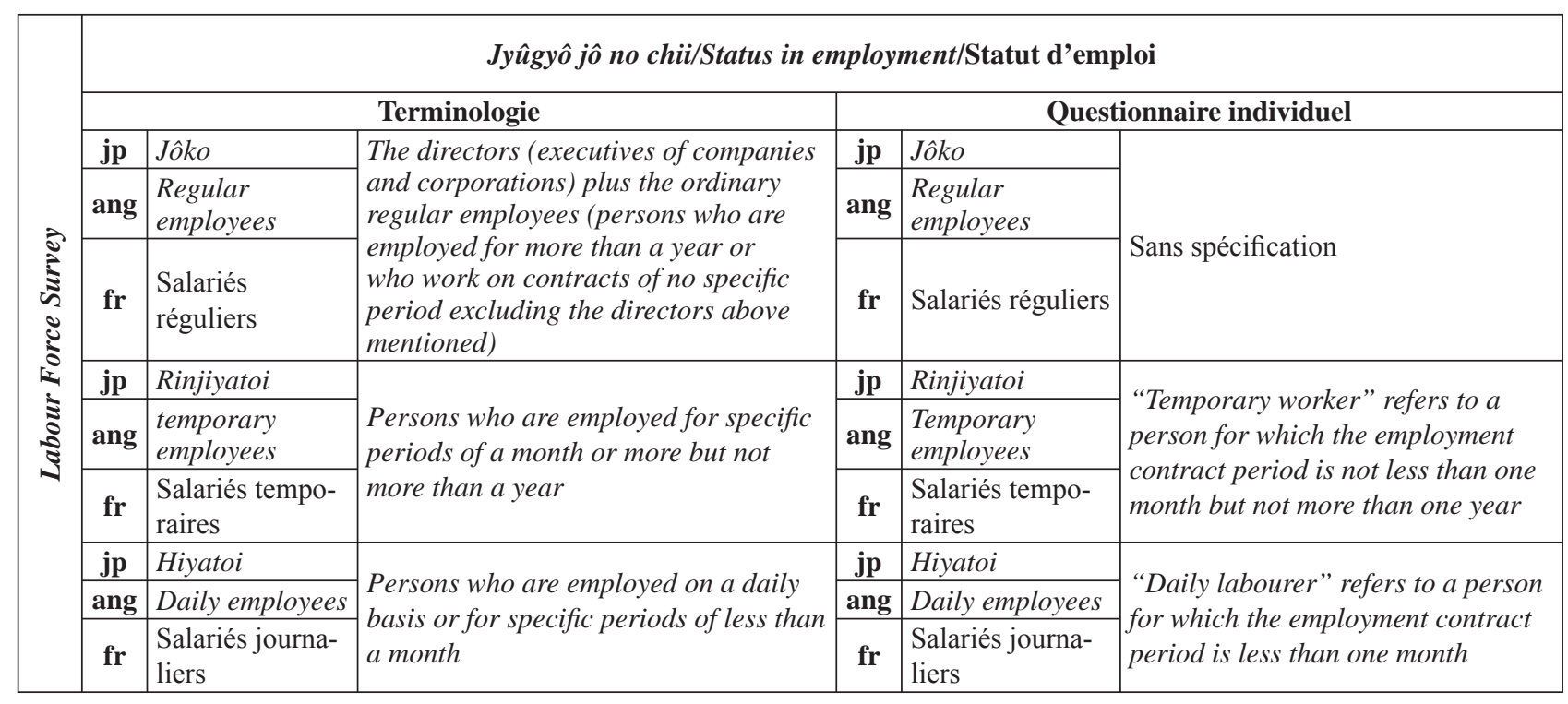

\begin{tabular}{|c|c|c|c|c|c|c|}
\hline \multirow{11}{*}{ 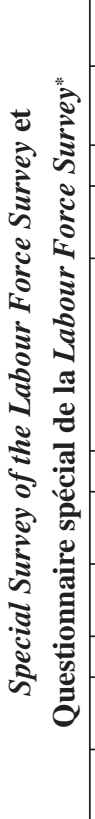 } & \multicolumn{6}{|c|}{ Jyûgyô jô no chii/Status in employment/Statut d'emploi } \\
\hline & \multicolumn{3}{|r|}{ Terminologie } & \multicolumn{3}{|c|}{$\begin{array}{l}\text { Questionnaire individuel et manuel d'aide } \\
\text { pour remplir le questionnaire }\end{array}$} \\
\hline & jp & Jôko & \multirow{3}{*}{$\begin{array}{l}\text { Executives of company/corporation } \\
\text { (Executives, managing directors } \\
\text { and auditors of private companies, } \\
\text { associations, public corporations, etc.) } \\
\text { and ordinary employees, ippan jôko, } \\
\text { (persons who work on contracts of no } \\
\text { specific period or a year or more of } \\
\text { employment excluding executives of } \\
\text { company/corporation above mentioned }\end{array}$} & jp & Jôko & \multirow[b]{3}{*}{$\begin{array}{l}\text { Personne employée pour une durée } \\
\text { d'un an et plus, ou qui travaillent sur } \\
\text { un contrat à durée indéterminée }\end{array}$} \\
\hline & ang & $\begin{array}{l}\text { Regular } \\
\text { employees }\end{array}$ & & ang & $\begin{array}{l}\text { Regular } \\
\text { employee }\end{array}$ & \\
\hline & fr & $\begin{array}{l}\text { Salariés } \\
\text { réguliers }\end{array}$ & & fr & Salarié régulier & \\
\hline & jp & Rinjiyatoi & \multirow{3}{*}{$\begin{array}{l}\text { Persons who work on contracts of a } \\
\text { month or more but not more than a year }\end{array}$} & jp & Rinjiyatoi & \multirow{3}{*}{$\begin{array}{l}\text { Personne employée pour une durée } \\
\text { d'un an et plus, ou qui travaillent sur } \\
\text { un contrat à durée indéterminée }\end{array}$} \\
\hline & ang & $\begin{array}{l}\text { Temporary } \\
\text { employees }\end{array}$ & & ang & $\begin{array}{l}\text { Temporary } \\
\text { employee }\end{array}$ & \\
\hline & fr & $\begin{array}{l}\text { Salariés } \\
\text { temporaires }\end{array}$ & & fr & $\begin{array}{l}\text { Salarié } \\
\text { temporaire }\end{array}$ & \\
\hline & jp & Hiyatoi & \multirow{3}{*}{$\begin{array}{l}\text { Persons who work on a daily basis or on } \\
\text { contracts of less than a month }\end{array}$} & jp & Hiyatoi & \multirow{3}{*}{$\begin{array}{l}\text { Personne employée sur une base } \\
\text { journalière ou pour une durée } \\
\text { déterminée inférieure à un mois }\end{array}$} \\
\hline & ang & $\begin{array}{l}\text { Daily } \\
\text { employees }\end{array}$ & & ang & Daily labourer & \\
\hline & $\mathbf{f r}$ & $\begin{array}{l}\text { salariés } \\
\text { journaliers }\end{array}$ & & $\mathbf{f r}$ & Salarié journalier & \\
\hline
\end{tabular}

* Aucune spécification quant à la koyô keitai, forme d'emploi, dans la SSLFS. 


\begin{tabular}{|c|c|c|c|c|c|c|}
\hline \multirow{20}{*}{ 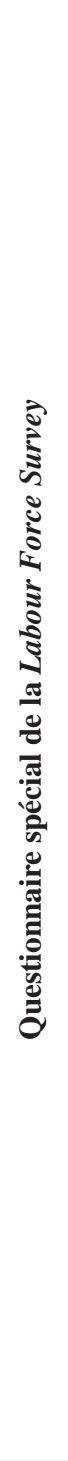 } & \multicolumn{6}{|c|}{ Koyô keitai/Form of employment/forme d'emploi (According to how their position is called at their workplace) } \\
\hline & \multicolumn{3}{|c|}{ Terminologie } & \multicolumn{3}{|c|}{ Manuel d'aide pour remplir le questionnaire } \\
\hline & jp & $\begin{array}{l}\text { Seiki no shokuin, } \\
\text { jyûgyôin }\end{array}$ & \multirow{3}{*}{$\begin{array}{l}\text { Sans } \\
\text { spécification }\end{array}$} & jp & \begin{tabular}{|l} 
Seiki no shokuin, \\
jyûgyôin
\end{tabular} & \multirow{3}{*}{$\begin{array}{l}\text { S'applique aux personnes appelées ippan shokuin } \\
\text { (salarié ordinaire) ou seishain (salarié titulaire), etc. }\end{array}$} \\
\hline & ang & Regular staff & & ang & Regular employee & \\
\hline & fr & Salarié régulier & & fr & Salarié régulier & \\
\hline & jp & Pâto & \multirow{3}{*}{$\begin{array}{l}\text { Sans } \\
\text { spécification }\end{array}$} & jp & Pâto & \multirow{6}{*}{$\begin{array}{l}\text { S'appliquent aux personnes qui sont appelées ainsi } \\
\text { ou d'une manière similaire sur le lieu de travail sans } \\
\text { qu'on tienne compte de leur horaire de travail ni du } \\
\text { nombre de jours travaillés }\end{array}$} \\
\hline & ang & Part-time worker & & ang & Part-time worker & \\
\hline & $\mathrm{fr}$ & \begin{tabular}{|l|} 
Travailleur à \\
temps partiel
\end{tabular} & & $\mathbf{f r}$ & \begin{tabular}{|l|} 
Travailleur à \\
temps partiel
\end{tabular} & \\
\hline & jp & Arubaito & \multirow{3}{*}{$\begin{array}{l}\text { Sans } \\
\text { spécification }\end{array}$} & jp & Arubaito & \\
\hline & \begin{tabular}{|l|} 
ang \\
\end{tabular} & Arubaito & & ang & Arubaito & \\
\hline & $\mathrm{fr}$ & Arubaito & & fr & Arubaito & \\
\hline & jp & $\begin{array}{l}\text { Rôdôsha haken } \\
\text { jigyôsho no haken } \\
\text { shain }\end{array}$ & \multirow{3}{*}{$\begin{array}{l}\text { Sans } \\
\text { spécification }\end{array}$} & jp & $\begin{array}{l}\text { Jinzai haken kigyô } \\
\text { no haken shain }\end{array}$ & \multirow{3}{*}{$\begin{array}{l}\text { S'applique aux personnes envoyées par une agence } \\
\text { de travail intérimaire dans le cadre du Worker } \\
\text { Dispatching Law. Si votre cas correspond à cette } \\
\text { catégorie, choisissez celle-ci même si vous êtes } \\
\text { appelé par un autre terme listé ici }\end{array}$} \\
\hline & ang & $\begin{array}{l}\text { Dispatched } \\
\text { workers from } \\
\text { temporary labour } \\
\text { agency }\end{array}$ & & ang & $\begin{array}{l}\text { Worker dispatched } \\
\text { from a dispatching } \\
\text { service agency }\end{array}$ & \\
\hline & fr & $\begin{array}{l}\text { Intérimaire envoyé } \\
\text { par l'agence de } \\
\text { travail intérimaire } \\
\end{array}$ & & $\mathrm{fr}$ & $\begin{array}{l}\text { Intérimaire envoyé } \\
\text { par l'agence de } \\
\text { travail intérimaire }\end{array}$ & \\
\hline & jp & $\begin{array}{l}\text { Keiyaku shain, } \\
\text { shokutaku }\end{array}$ & \multirow{3}{*}{$\begin{array}{l}\text { Sans } \\
\text { spécification }\end{array}$} & jp & \begin{tabular}{|l}
$\begin{array}{l}\text { Keiyaku shain, } \\
\text { shokutaku }\end{array}$ \\
\end{tabular} & \multirow{3}{*}{$\begin{array}{l}\text { Contractuel s'applique aux personnes qui sont } \\
\text { engagées avec un contrat à durée déterminée pour } \\
\text { occuper un poste à une compétence spécifique. } \\
\text { Shokutaku s'applique aux personnes appelées ainsi } \\
\text { ou de manière similaire sur leur lieu de travail, sans } \\
\text { tenir compte des conditions de travail ou de la durée } \\
\text { du contrat }\end{array}$} \\
\hline & ang & $\begin{array}{l}\text { Contract employee } \\
\text { or entrusted } \\
\text { employee }\end{array}$ & & ang & $\begin{array}{l}\text { Contract employee } \\
\text { or entrusted } \\
\text { employee }\end{array}$ & \\
\hline & fr & $\begin{array}{l}\text { Salarié contractuel } \\
\text { ou salarié à qui on } \\
\text { confie le travail }\end{array}$ & & fr & $\begin{array}{l}\text { Salarié contractuel } \\
\text { ou salarié à qui on } \\
\text { confie le travail }\end{array}$ & \\
\hline & jp & Sonota & \multirow{3}{*}{$\begin{array}{l}\text { Sans spécifi- } \\
\text { cation }\end{array}$} & jp & Sonota & \multirow{3}{*}{ Sans spécification } \\
\hline & ang & Other & & ang & Other & \\
\hline & fr & Autres & & fr & Autres & \\
\hline
\end{tabular}

\begin{tabular}{|c|c|c|c|c|c|c|}
\hline \multirow{11}{*}{ 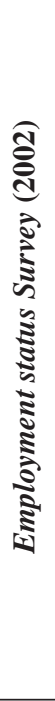 } & \multicolumn{6}{|c|}{ Jyûgyô jô no chii/Status in employment/Statut d'emploi } \\
\hline & \multicolumn{3}{|r|}{ Terminologie } & \multicolumn{3}{|r|}{ Questionnaire individuel } \\
\hline & jp & Ippan jôko & \multirow{3}{*}{$\begin{array}{l}\text { Employees other than executives, } \\
\text { "Temporary employees" and } \\
\text { "Daily employees" }\end{array}$} & jp & Jôko & \multirow{3}{*}{ Sans spécification } \\
\hline & ang & $\begin{array}{l}\text { Ordinary } \\
\text { employees }\end{array}$ & & ang & $\begin{array}{l}\text { Ordinary } \\
\text { employee }\end{array}$ & \\
\hline & fr & \begin{tabular}{|l|}
$\begin{array}{l}\text { Salariés } \\
\text { réguliers }\end{array}$ \\
\end{tabular} & & $\mathbf{f r}$ & \begin{tabular}{|l|}
$\begin{array}{l}\text { Salarié } \\
\text { régulier }\end{array}$ \\
\end{tabular} & \\
\hline & jp & Rinjiyatoi & \multirow{3}{*}{$\begin{array}{l}\text { Those who are employed on a } \\
\text { contract of employment for a term } \\
\text { of a month or more but less than } \\
\text { a year }\end{array}$} & jp & Rinjiyatoi & \multirow{6}{*}{$\begin{array}{l}\text { Temporary employee» means a worker with an } \\
\text { employment contract of } 1-12 \text { months. "Daily } \\
\text { employee" means a worker with an employ- } \\
\text { ment contract of less than a month. }\end{array}$} \\
\hline & ang & $\begin{array}{l}\text { Temporary } \\
\text { employees }\end{array}$ & & ang & \begin{tabular}{|l} 
Temporary \\
employee
\end{tabular} & \\
\hline & fr & \begin{tabular}{|l|} 
Salariés \\
temporaires
\end{tabular} & & fr & \begin{tabular}{|l|} 
Salarié \\
temporaire
\end{tabular} & \\
\hline & jp & Hiyatoi & \multirow{3}{*}{$\begin{array}{l}\text { Those who are employed on a } \\
\text { daily basis or on a contract of } \\
\text { employment for a term of less than } \\
\text { a month. }\end{array}$} & jp & Hiyatoi & \\
\hline & ang & \begin{tabular}{|l|}
$\begin{array}{l}\text { Daily } \\
\text { employees }\end{array}$ \\
\end{tabular} & & ang & \begin{tabular}{|l|}
$\begin{array}{l}\text { Daily } \\
\text { employee }\end{array}$ \\
\end{tabular} & \\
\hline & fr & \begin{tabular}{|l|}
$\begin{array}{l}\text { Salariés } \\
\text { journaliers }\end{array}$ \\
\end{tabular} & & fr & \begin{tabular}{|l|}
$\begin{array}{l}\text { Salarié } \\
\text { journalier }\end{array}$ \\
\end{tabular} & \\
\hline
\end{tabular}




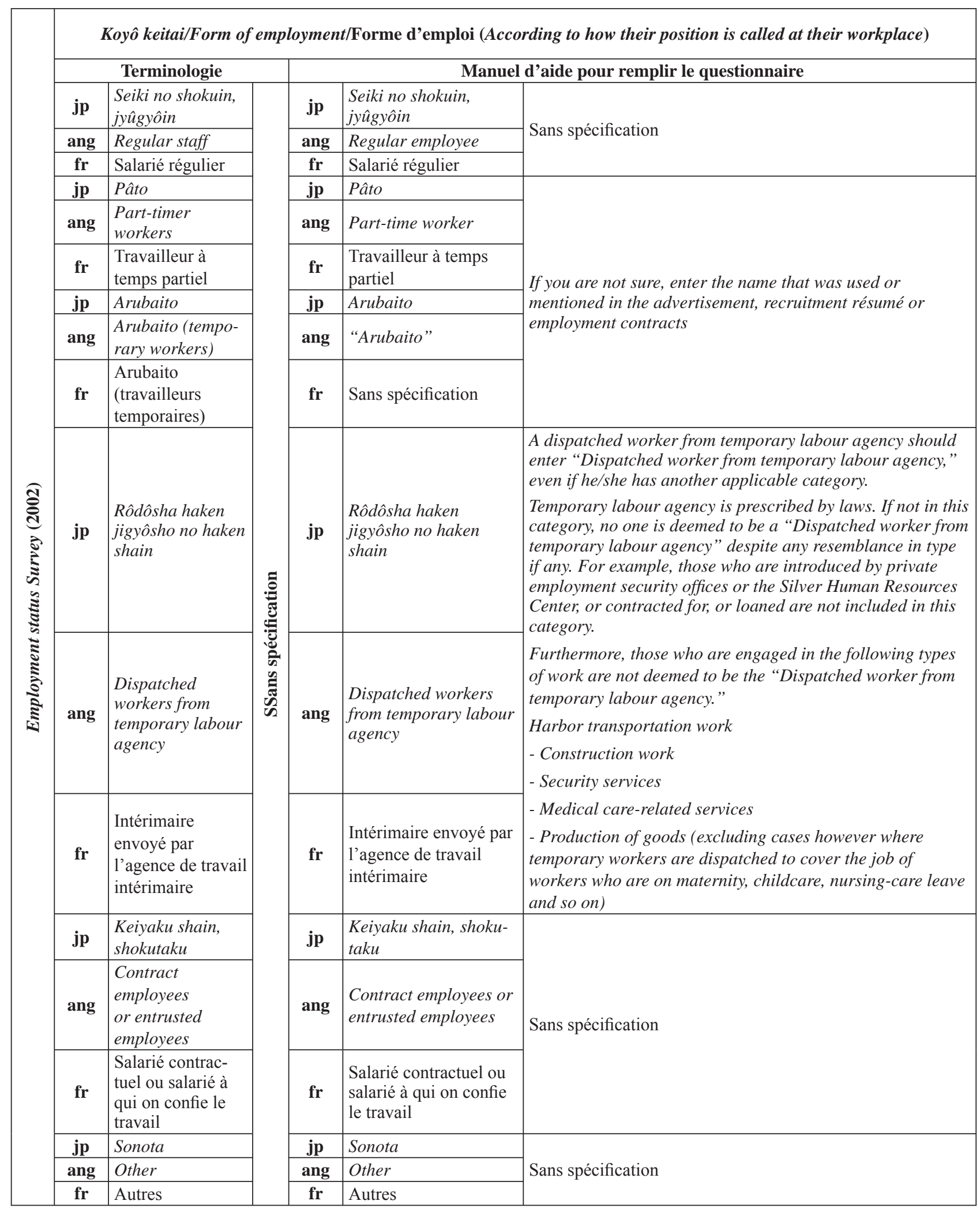

